\title{
PITCH TRACKING FOR MODEL-BASED SPEECH SEPARATION
}

\author{
S. W. Lee ${ }^{1}$, Frank K. Soong ${ }^{2}$, P. C. Ching ${ }^{1}$ and Tan Lee ${ }^{1}$ \\ ${ }^{1}$ Department of Electronic Engineering, The Chinese University of Hong Kong, Hong Kong SAR \\ ${ }^{2}$ Microsoft Research Asia, Beijing
}

\begin{abstract}
Estimating multiple pitch frequencies of concurrent speech sources from a single-microphone input is essential to speech separation. Nevertheless, pitch cues of individual sources are weakened by each other, making the estimation unreliable. This paper presents a pitch tracking method that incorporated in a model-based separation framework. Multiple pitch estimation is simplified into single pitch estimation by segregating the source envelope from mixture spectrum with statistics of familiar speech patterns. Comprehensive experiments have compared the proposed tracking method with a recently reported multiple pitch estimator and its modified version equipped with ideal pitch cues. Lower estimation errors are achieved. Furthermore, this approach is applicable to other model-based frameworks as well. ${ }^{1}$
\end{abstract}

Index Terms - pitch tracking, speech separation, speech modeling, vocal tract, harmonics

\section{INTRODUCTION}

Speech signal comprises voiced and unvoiced sounds. Voiced speech is produced by the vocal folds vibration which generates a periodic airflow to excite the vocal tract filter. This is where the sensation of pitch (tone) originates. The more rapid the oscillation, the higher is the pitch. Furthermore, voiced speech possesses regular patterns in both waveform and frequency spectrum. By measuring the inverse of the period of the waveform, we obtain the fundamental frequency (F0) that characterizes the pitch.

Pitch estimation has been a long-lasting research topic, from single pitch tracking in speech coding [1] to multiple pitch (multipitch) tracking in speech source segregation and music information retrieval [2], [3]. It is also useful for automatic speech recognition of tonal languages like Mandarin [4] and hearing prostheses [5]. The majority of pitch tracking methods aims at robust estimation of a single pitch track (for example, [6], [7]). Autocorrelation function of an input signal is generally utilized to locate the pitch period and statistics of continuous pitch dynamics and transitions between voiced and unvoiced states are employed to improve the robustness. Similar features are utilized in multipitch tracking. Nevertheless, multipitch tracking is intrinsically difficult. As pitch cues of multiple speech sources are simultaneously present, they are often weakened by each other and may be occasionally degenerated into a single pitch. More sophisticated techniques, such as auditory processing, iterative

\footnotetext{
${ }^{1}$ This work is partially supported by a grant awarded by the Hong Kong Research Grants Council. The work is also affiliated with the CUHK MoE-Microsoft Key Laboratory of Human-Centric Computing and Interface Technologies.
}

estimation-subtraction [8] and multipitch hypotheses [9], are applied to combat the interference from competing speech.

In this paper we focus on pitch tracking for speech separation in a single-microphone input. Human being is capable of segregating interested sound sources from interference and background noise [10]. Our perceptual system performs separation by going over primitive, acoustic regularities (for example, harmonicity and continuity etc.) and applying any relevant knowledge like familiar speech patterns. Pitch information is always exploited to represent harmonicity [11], [12]. Power of the observation spectrum is divided between sources accordingly. Alternatively, comb filtering can be used to extract or suppress the interfering source.

Accurate pitch information is essential for both speech separation, as well as generation of a sound source. One of the two key elements of a speech source is the harmonic structure, which is basically governed by the F0. Recently, we have proposed a speech separation algorithm based on speech production and modeling of familiar speech patterns [13]. It estimates a target speech source in terms of its spectral envelope trajectory and harmonic structure. Hence, pitch tracking is necessary and influential to the separation performance.

In [13], the algorithm and experiments on separation, particularly on spectral envelope trajectory, were reported. This paper presents the role of pitch harmonics in model-based speech separation and a pitch estimation algorithm. Comprehensive experiments on different speech materials including continuous Chinese utterances were carried out. The evaluation on pitch tracking performance shows that better pitch estimation (in terms of fine detection error, gross detection error and pitch number detection error rate) is achieved. Moreover, some fundamental difficulties in multipitch tracking are bypassed, which is beneficial to general model-based separation or enhancement methods.

\section{PITCH HARMONICS AND MODEL-BASED SPEECH SEPARATION}

This section briefly illustrates a recently proposed model-based speech separation and describes the role of pitch harmonics. An input mixture signal $x(n)$ is related to its two speech sources $x_{1}(n)$ and $x_{2}(n)$ as

$$
x(n)=x_{1}(n)+x_{2}(n)
$$

By source-filter modeling, a speech signal is modeled as the output of passing an excitation source through a vocal tract filter. Having these two elements enables the construction of this speech signal. In our model-based speech separation [13], target speech sources are estimated in a 'synthetic' manner, by working out the associated spectral envelope, voicing information and the harmonic structure, as depicted in Fig. 1. Let $x_{i}(n)$ be the estimate for source $i(i \in[1,2])$. 


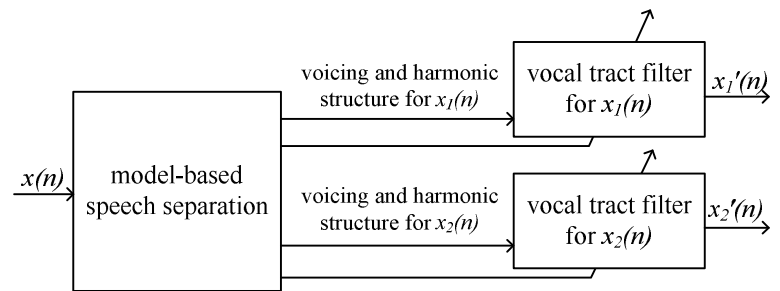

Fig. 1. Basic idea of the model-based speech separation.

The separation algorithm is divided into two stages. The block-diagram of the model-based speech separation system is shown in Fig. 2. The first stage is for the envelope part. For each individual target source $x_{i}(n)$, the mixture spectrum $X(\omega, t)$ is cleaned up to approximate the target spectral envelope $X_{i}(\omega, t)$. $X(\omega, t)$ and $X_{i}(\omega, t)$ denote the frequency spectra for an input mixture frame and a target source frame respectively.

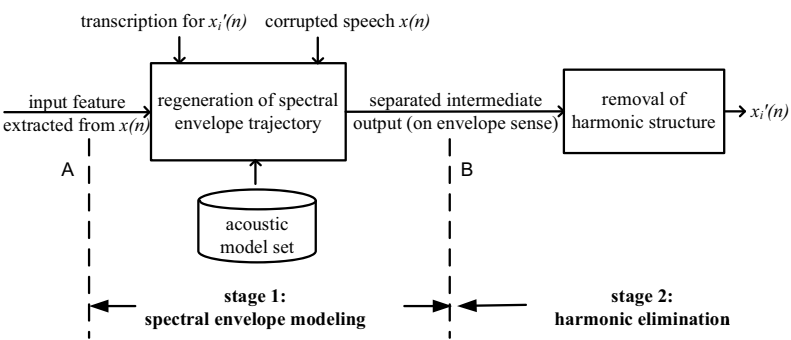

Fig. 2. Block-diagram of the model-based separation system.

The input feature extracted from mixture $x(n)$ is compared with a set of familiar speech models (acoustic models) that trained beforehand. This is accomplished by speech recognition and the alignment of best matched speech models is output. In the current setup, it is assumed that the text transcription for both source signals is available. Otherwise, robust recognition would be critical. The 'model-based' idea here is twofold: source-filter modeling and speech acoustic models. The aligned model parameters dictate the spectral envelope trajectory of the target speech source. It is further enhanced to give a continuous trajectory with an appropriate power level, according to the dynamic statistics of spectrum collected and mixture spectrum $X(\omega)$ t). Wiener filtering is finally employed to explicitly remove the interfering source $x_{j}(n)(j \neq i)$, and retain vocal tract properties associated with $x_{i}(n)$.

The second stage focuses on pitch harmonics. Harmonic structures of both $x_{1}(n)$ and $x_{2}(n)$ remain after the first stage. Irrelevant harmonics which belong to the interfering source should be removed. Comb filtering in the form of pitch prediction error filter [1] is incorporated. Fig. 3 shows the block-diagram of the second stage. Comb filtering is applied for frames where there is harmonic structure from interfering source. Otherwise, an all-pass filter is used. The impulse response of a pitch prediction error filter is given by

$$
\begin{aligned}
h_{p}(n) & =\delta(n)-\beta_{1} \delta(n-L) \\
& -\beta_{2} \delta(n-(L+1))-\beta_{3} \delta(n-(L+2))
\end{aligned}
$$

where $\beta_{j}$ are the filter coefficients for $j \in[1,2,3]$. $L$ is the pitch lag of the interfering source. Notches are found at the respective harmonic frequencies in the power spectrum of $h_{p}(n)$ (as shown in the comb filter in Fig. 3). Hence, the remaining pitch harmonics of the interfering source are removed. Subsequently, the output is a single speech source with the associated properties of vocal tract and pitch harmonics.

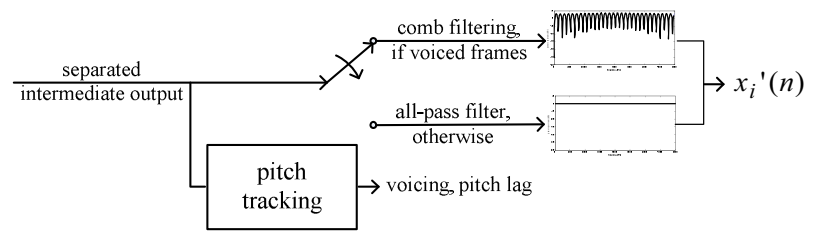

Fig. 3. Block-diagram of stage 2: harmonic elimination.

\section{PITCH TRACKING}

Our pitch estimation is based on a robust multipitch tracking algorithm by $\mathrm{Wu}$ et al. [9], but revised to exploit the design of the model-based separation framework. Wu's tracking algorithm has been reported to give better performance over other existing methods under various conditions. The complete pitch tracking system consists of four major processes, as depicted in Fig. 4.

Multichannel front-end: Pitch values of individual sources are extracted based on the statistical anticipation on normalized correlograms (correlation coefficient functions) computed at a set of cochlear channels. Specifically, the delay lag giving the maximum correlation coefficient indicates the period of a voiced segment. Moreover, pitch period in human speech tends to fluctuate slightly over adjacent periods [7]. For each pitch hypothesis, the closer the delay lag to the ideal pitch lag, the more probable the pitch hypothesis is. By measuring the difference $\delta$ between the ideal pitch lag and the closest peak in the correlation coefficient function, the contribution of a channel to a given pitch hypothesis is evaluated.

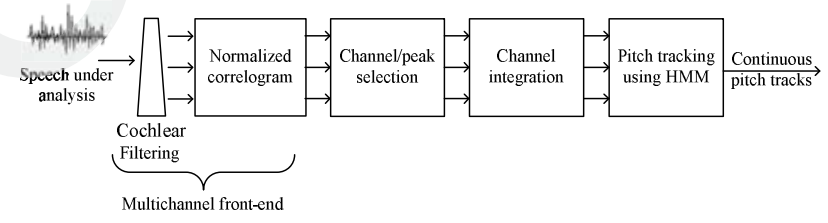

Fig. 4. Block-diagram of the multipitch tracking algorithm (revised from [9]).

Channel/peak selection: As the mixture input $x(n)$ contains multiple speech sources, peaks in correlation coefficients may come from the interfering source, rather than the target source. Reliable channels and peaks are identified by inspecting the correlation coefficient function.

Channel integration: With the set of selected peaks, channel contribution for a given pitch hypothesis is evaluated by the Laplacian modeling of $\delta$ and then integrated over all channels into a summary score. Similarly, the hypothesis of two pitch lags is formulated by assuming that one pitch lag is stronger and considering all the possible permutations of pitch lag pair.

Pitch tracking using HMM: Continuous pitch tracks are finally generated by using a hidden Markov model (HMM). Consecutive time frames are connected by pitch dynamics and optimal pitch tracks are extracted by the Viterbi algorithm. Two aspects of pitch dynamics are incorporated. They are dynamics within a continuous pitch segment and transition between different numbers of pitch sources.

Referring to our separation framework (Fig. 2), pitch tracking can be performed before the first stage as multipitch tracking (at point A) or after the first stage as single pitch tracking (at point $\mathrm{B}$ ). 
Single pitch tracking is simpler and more accurate than multipitch tracking. By exploiting the design of the model-based separation and statistics of familiar speech sources, the above multipitch tracking algorithm is revised to operate after the first stage (at B). The separated intermediate output after envelope regeneration is consequently utilized to provide a cleaner input for estimation, simplifying a multipitch tracking problem into a single pitch tracking one and bypassing some of the estimation difficulties from mixture signals.

\section{EXPERIMENTS AND DISCUSSIONS}

\begin{tabular}{|c|c|}
\hline category & speech data \\
\hline $\begin{array}{l}\text { I } \\
\text { (verifi- } \\
\text { cation } \\
\text { purpose) }\end{array}$ & $\begin{array}{l}\text { English speech source utterances and mixtures used in } \\
\text { [9], so as to verify the implementation of original Wu's } \\
\text { multipitch tracking; } \\
\text { Half of the source utterances are fully voiced (specially- } \\
\text { designed speech) and at least one source utterance in } \\
\text { mixture signals is fully voiced; } \\
1711 \text { frames in total; } \\
\text { Reference pitch contours are obtained by pitch tracking } \\
\text { from source utterances first and then manual correction }\end{array}$ \\
\hline II & $\begin{array}{l}\text { Clean continuous Chinese speech utterances with } \\
\text { typical, intermittent voiced/unvoiced transitions; } \\
1056 \text { frames in total; } \\
\text { Reference pitch contours are derived by laryngographs }\end{array}$ \\
\hline III & $\begin{array}{l}\text { Mixture of continuous Chinese speech utterances (made } \\
\text { by category II data at signal-to-interference ratio } 0 \mathrm{~dB} \text { ); } \\
1056 \text { frames in total }\end{array}$ \\
\hline
\end{tabular}

Table 1. Speech data used for performance evaluation of pitch estimation.

The revised pitch estimation is evaluated on different sets of speech materials. Table 1 lists out the speech data used. The performance is quantitatively assessed by measuring three types of pitch estimation errors: gross detection error $\left(E_{\text {gross }}\right)$, fine detection error $\left(E_{f i n e}\right)$ and pitch number detection error rate $\left(E_{m n}\right)$. Given that pitch frequency deviation $(\Delta f)$ is calculated by

$$
\Delta f=\frac{\left|F 0^{\prime}-F 0\right|}{F 0} \times 100 \%
$$

where $F 0$ is the reference pitch frequency and $F 0^{\prime}$ is the estimated pitch frequency. $E_{\text {gross }}$ refers to the percentage of frames where $\Delta f$ is larger than $20 \%$. $E_{\text {fine }}$ is defined as the average $\Delta f$ for frames without gross detection error. $E_{m n}$ measures the error rate of frames where a $m$-pitch frame (a frame containing $m$ voiced sources) is wrongly classified as a $n$-pitch frame. A total gross error is further indicated by

$$
E_{\text {total }}=E_{\text {gross }}+\sum_{\{\forall(m, n)\}} E_{m n}
$$

The implementation of Wu's method is first verified by inspecting the measured error rates of Category I mixture data and output pitch tracks. The results of single pitch tracking of source utterances are also checked.

Category II: One example of output pitch tracks is shown in Fig. 5. The pitch estimates within voiced segments are accurate. Nevertheless, estimates at segment boundaries are slightly deviated and the voicing decisions are sometimes wrongly determined. The error measurements are shown in Table 2.

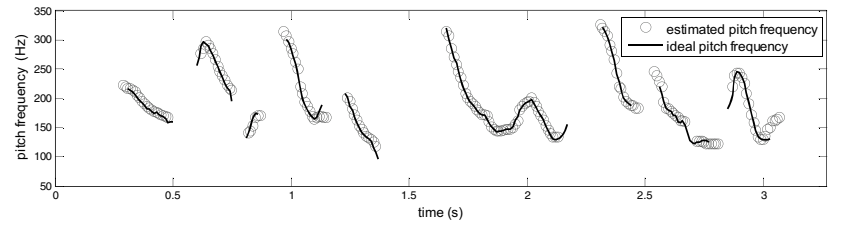

Fig. 5. Output pitch track from an utterance in Category II.

\begin{tabular}{|c|c|c|c|c|}
\hline $\begin{array}{c}\text { Error } \\
\text { type }\end{array}$ & $\begin{array}{c}\text { Category } \\
\text { II }\end{array}$ & $\begin{array}{c}\text { Category III } \\
\text { estimated at A }\end{array}$ & $\begin{array}{c}\text { Category III } \\
\text { control } \\
\text { estimated at A }\end{array}$ & $\begin{array}{c}\text { Category III } \\
\text { estimated at B }\end{array}$ \\
\hline$E_{\text {fine }}$ & 3.317 & 4.53 & 2.669 & 2.428 \\
\hline$E_{\text {total }}$ & 34.325 & 150.273 & 76.012 & 50.608 \\
\hline$E_{\text {gross }}$ & 0 & 16.901 & 2.564 & 2.706 \\
\hline$E_{01}$ & 26.712 & 25.074 & 0.926 & 29.274 \\
\hline$E_{02}$ & - & 14.815 & 0 & - \\
\hline$E_{10}$ & 7.613 & 13.675 & 25.641 & 18.629 \\
\hline$E_{12}$ & - & 0.855 & 15.385 & - \\
\hline$E_{20}$ & - & 1.575 & 3.937 & - \\
\hline$E_{21}$ & - & 77.378 & 27.559 & - \\
\hline
\end{tabular}

Table 2. Error measurements (in percentage), where '-' denotes 'not applicable'.

Category III estimated at A: Fig. 6 shows an example of two pitch contours estimated. The estimates are fragmentary. There are occasionally missing pitch values at the middle of voiced segments. Pitch values estimated, however, remain closed to the reference values. Estimates from one source are easily assigned to the other source, since the two pitch contours cross. From the error measurements, the performance of multipitch tracking is unsatisfactory and worse than single pitch tracking.

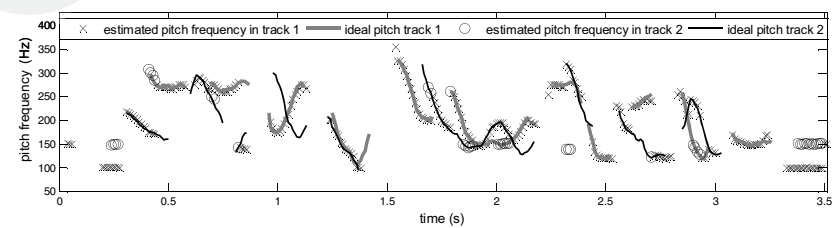

Fig. 6. Output pitch tracks of Category III data, estimated at A.

Category III control estimated at A: This control experiment studies the upper bound performance of multipitch tracking for Category III data. Peaks in correlograms belong to ideal pitch periods are kept and all relevant parameters, such as transition probabilities, are tuned with a small amount of Category III data. This simulates a correlogram with correct peaks of both sources. The error measurements are given in Table 2. By keeping all peaks associated with the true pitch lags, $E_{\text {fine }}, E_{\text {gross }}, E_{01}, E_{02}$ and $E_{21}$ are largely reduced. The performance of the control experiment of Category III data is better than standard multipitch tracking, but still far away from single pitch tracking (Category II). The advantages of single pitch tracking are clearly shown.

Category III estimated at B: We propose to perform pitch estimation for separated intermediate outputs at B, so as to imitate single pitch tracking. Consequently, the observed correlogram peaks probably come from the target source, thus avoiding the influence of interference peaks. The pitch assignment (permutation) problem is also bypassed. All relevant parameters are tuned again. The error measurements and a set of pitch tracks are shown in Table 2 and Fig. 7 respectively. 

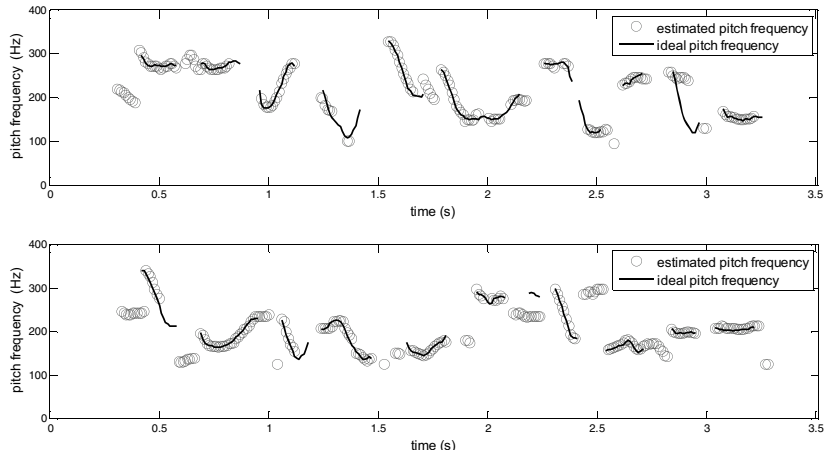

Fig. 7. A set of output pitch tracks estimated at B from a Category III mixture signal.

Compared with the errors of Category III estimated at A, better performance is obtained from pitch tracking at B (that is, after envelope regeneration). $E_{\text {fine }}$ and $E_{\text {total }}$ are lower. They are also lower than the one obtained from the Category III control experiment. Hence, the revised pitch tracking after envelope regeneration provides better estimation than multipitch tracking at the beginning of the separation framework. Compared with the performance on clean speech sources (Category II), the proposed approach has a higher $E_{\text {total }}$, but a bit lower $E_{\text {fine }}$. The increase in $E_{\text {total }}$ mainly come from $E_{10}$, which accounts for over $65 \%$ increase in $E_{\text {total }}$. This reflects that one-pitch frames are sometimes misclassified as zero-pitch frames. One possible reason is that the signals obtained after envelope regeneration are not absolutely clean. There is still some residual of the interfering source, contaminating the channel and peak selection.

This pitch tracking after envelope regeneration approach is equally applicable to other frameworks, such as [14]. The fragment decoder determines the set of time-frequency units (T-F mask) that belong to a target speech source, having a similar functionality as the first stage of our model-based separation algorithm. Pitch estimation with a T-F mask enables single pitch tracking for individual sources, with better estimation performance over multipitch tracking.

\section{CONCLUSIONS}

Multitpitch tracking is difficult per se, but indispensible to speech separation. This paper presents a pitch tracking method adopted in a recently proposed model-based separation algorithm. The role of pitch harmonics is first studied, from the perspectives of separation and source reconstruction. A multipitch tracking method is revised to utilize the separated intermediate output after envelope estimation. This essentially simplifies the problem to single pitch tracking and this is applicable to other frameworks with T-F masks as well. Experimental results have shown that the proposed pitch tracking approach leads to lower pitch estimation errors over standard multipitch tracking and the best performance of multipitch tracking with ideal correlogram.

\section{REFERENCES}

[1] R. P. Ramachandran and P. Kabal, "Pitch prediction filters in speech coding," IEEE Trans. Acoust., Speech, Signal Process., vol. 37, no. 4, pp. 467-478, Apr. 1989

[2] S. W. Lee, F. K. Soong, and P. C. Ching, "Harmonic filtering for joint estimation of pitch and voiced source with single- microphone input," in Proc. Eurospeech, Lisbon, Sep. 2005, pp. 309-312.

[3] H. Kameoka, T. Nishimoto, and S. Sagayama, "A multipitch analyzer based on harmonic temporal structured clustering," IEEE Trans. Acoust., Speech, Language Process., vol. 15, no. 4, pp. 982994, Apr. 2007.

[4] T. Lee and Y. Qian, "Tone modeling for speech recognition," in Advances in Chinese Spoken Language Processing, C.-H. Lee, H. Li, L.-S. Lee, R.-H. Wang, and Q. Huo, Eds. Singapore: World Scientific Publishing Co. Pte. Ltd., 2007, ch. 8, pp. 179-200.

[5] S. M. Rosen, A. J. Fourcin, and B. C. J. Moore, "Voice pitch as an aid to lipreading," Nature, vol. 291, no. 5811, pp. 150-152, May 1981.

[6] D. Talkin, "A robust algorithm for pitch tracking (RAPT)," in Speech Coding and Synthesis, W. B. Kleijn and K. K. Paliwal, Eds. Amsterdam, The Netherlands: Elsevier Science B.V., 1995, ch. 14, pp. 495-518.

[7] W. J. Hess, Pitch Determination of Speech Signals: Algorithms and Devices. New York: Springer, 1983.

[8] A. P. Klapuri, "Multiple fundamental frequency estimation based on harmonicity and spectral smoothness," IEEE Trans. Speech Audio Process., vol. 11, no. 6, pp. 804-816, Nov. 2003.

[9] M. Wu, D. Wang, and G. J. Brown, "A multipitch tracking algorithm for noisy speech," IEEE Trans. Speech Audio Process., vol. 11, no. 3, pp. 229-241, May 2003.

[10] A. S. Bregman, Auditory Scene Analysis: The Perceptual Organization of Sound. Cambridge, Massachusetts: The MIT Press, 1990

[11] G. J. Brown and M. Cooke, "Computational auditory scene analysis," Computer Speech and Language, vol. 8, no. 4, pp. 297336, Oct. 1994.

[12] G. Hu and D. Wang, "Monaural speech segregation based on pitch tracking and amplitude modulation," IEEE Trans. Neural Netw., vol. 15, no. 5, pp. 1135-1150, Sep. 2004.

[13] S. W. Lee, F. K. Soong, and P. C. Ching, "Model-based speech separation with single-microphone input," in Proc. Interspeech, Antwerp, Aug. 2007, pp. 850-853.

[14] J. P. Barker, M. P. Cooke, and D. P. W. Ellis "Decoding speech in the presence of other sources," Speech Communication, vol. 45, no. 1, pp. 5-25, Jan. 2005. 